\title{
A case of imported Middle East Respiratory Syndrome coronavirus infection and public health response, Greece, April 2014
}

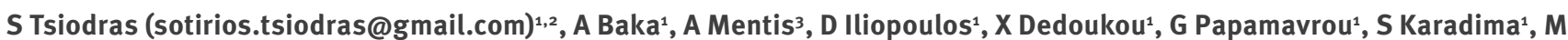
Emmanouil'3, A Kossyvakis's, N Spanakis ${ }^{4}$, A Pavli ${ }^{1}$, H Maltezou ${ }^{1}$, A Karageorgou ${ }^{5}$, G Spala ${ }^{1}$, V Pitiriga ${ }^{4}$, E Kosmas ${ }^{6}$, S Tsiagklis ${ }^{6}$, S Gkatzias $^{6}$, N G Koulouris 7 , A Koutsoukou ${ }^{8}$, P Bakakos ${ }^{7}$, E Markozanhs ${ }^{7}$, G Dionellis7, K Pontikis ${ }^{8}$, N Rovina ${ }^{8}$, M Kyriakopoulou ${ }^{8}$,

P Efstathiou ${ }^{5}$, T Papadimitriou ${ }^{1}$, J Kremastinou ${ }^{1}$, A Tsakris ${ }^{4}$, G Saroglou ${ }^{1,6}$

1. Hellenic Center for Disease Control and Prevention, Athens, Greece

2. University of Athens Medical School, Athens Greece

3. Hellenic Pasteur Institute, Athens, Greece

4. Microbiology Department, University of Athens Medical School, Athens, Greece

5. National Health Operations Center, Athens, Greece

6. Metropolitan Hospital Athens Greece

7. First Department of Respiratory Medicine, University of Athens Medical School, Athens, Greece

8. Intensive Care Medicine first Department of Respiratory Medicine, University of Athens Medical School, Athens Greece

Tsiodras S, Baka A, Mentis A, Iliopoulos D, Dedoukou X, Papamavrou G, Karadima S, Emmanouil M, Kossyvakis A, Spanakis N, Pavli A, Maltezou H, Karageorgou A, Spala G, Pitiriga V, Kosmas E, Tsiagklis S, Gkatzias S, Koulouris NG, Koutsoukou A, Bakakos P, Markozanhs E, Dionellis G, Pontikis K, Rovina N, Kyriakopoulou M, Efstathiou P, Papadimitriou T, Kremastinou J, Tsakris A, Saroglou G. A case of imported Middle East Respiratory Syndrome coronavirus infection and public health response, Greece, April 2014. Euro Surveill. 2014;19(16):pii=20782. Available online: http://www.eurosurveillance.org/ViewArticle.aspx?Articleld=20782

On 18 April 2014, a case of Middle East Respiratory Syndrome coronavirus (MERS-CoV) infection was laboratory confirmed in Athens, Greece in a patient returning from Jeddah, Saudi Arabia. Main symptoms upon initial presentation were protracted fever and diarrhoea, during hospitalisation he developed bilateral pneumonia and his condition worsened. During 14 days prior to onset of illness, he had extensive contact with the healthcare environment in Jeddah. Contact tracing revealed 73 contacts, no secondary cases had occurred by 22 April.

On 17 April 2014, the Hellenic Center for Disease Control and Prevention (HCDCP) in Athens Greece was notified about a suspected case of Middle East Respiratory Syndrome coronavirus (MERS-CoV) infection in a tertiary care hospital in Athens. The patient had presented a few hours after returning to Greece from Jeddah, Saudi Arabia. Herein we report preliminary findings of an extensive public health investigation conducted from 18 April 2014 (Good Friday) when the case was laboratory confirmed, through 22 April, when contact tracing was nearly complete.

\section{Case report}

A 69-year-old Greek national, who resides permanently in Jeddah, Saudi Arabia, presented to a tertiary care center with prolonged fever and diarrhoea, a few hours after arriving in Athens, Greece on 17 April 2014. He had travelled to Greece on the same day on two consecutive flights, one from Jeddah to Amman, Jordan and the other from Amman to Athens. Due to fear of contracting another disease during his travel, the patient was wearing a face mask for most of his journey to Athens up until reaching the hospital.

\section{Clinical presentation and laboratory findings in Athens}

He presented at the hospital in Athens with a fever of $38.3^{\circ} \mathrm{C}$ and low oxygen saturation (92\%). A chest x-ray depicted bilateral lung infiltrates consistent with viral pneumonia. The patient was immediately placed under isolation because of suspicion of MERS-CoV infection and treated by his physicians as community acquired pneumonia.

Laboratory tests were performed on the same day at the National Reference Laboratory for Influenza at the Hellenic Pasteur Institute, Athens. On 18 April, the MERS-CoV infection was confirmed, * in an oropharyngeal sample, based on real-time RT- PCR using primers for the upstream of envelope gene (UPE) as a screening test and for the open reading frame (ORF) $1 \mathrm{~A}$ gene as a confirmatory assay [1]. The same day, the diagnosis was confirmed at the Department of Microbiology of the University of Athens Medical School. Sequencing of the viral genome is ongoing. Laboratory testing for influenza virus, legionella and pneumococcus was negative as well as a stool culture for salmonella.*

After notification of the positive result for MERS-CoV, the patient was transferred to a specialised respiratory disease unit in the Chest Diseases Hospital of Athens where he was treated in a negative pressure room. His respiratory function gradually worsened and since 20 
April 2014, he is intubated and ventilated in critical condition in intensive care.

\section{Patient history in Saudi Arabia}

The case investigation was conducted through interviews with the patient (before his intubation) and his wife and was based on a standard form available by WHO [2]. Patient history revealed that on 8 April, while still in Jeddah, he had developed a fever and visited a local hospital, the Al-Jedaani Group of Hospitals. He returned to the hospital two days later, on 10 April, when he additionally developed diarrhoea and again on 14 April because of persisting symptoms. He was prescribed ciprofloxacin for a presumptive diagnosis of typhoid fever based on a single positive serology by Widal test ( $\mathrm{H}$-antigen test titer of 1:160). Tests for dengue fever and brucellosis were negative, no tests for MERS-CoV were performed. A chest $\mathrm{x}$-ray was negative for infiltrates.

The patient had additional extensive contact with the nosocomial environment in Jeddah from 31 March until 5 April, when his wife was hospitalised in the same hospital as above, with the laboratory confirmed diagnosis of typhoid fever (positive stool culture mentioned in the history of the patient without further documentation). The patient had continuous contact with his wife during her hospital stay. No MERS-CoV diagnostic was performed in the wife during her stay in the hospital in Jeddah.* Laboratory tests in blood, urine, and faeces for MERS-CoV in Greece, several days after her symptoms of infection had resolved, were negative.
The patient had further contact with the healthcare system between 25 and 29 March, when he accompanied his wife to her daily physiotherapy sessions at a rehabilitation center prior to her illness. A timeline of events is depicted in the figure.

The patient had no contact with camels, a known virus reservoir [3, 4] but reported indirect contact with bats, another possible reservoir of MERS-CoV [5], on 15 March outside of the incubation period for MERS-CoV, at an outdoor dinner. Moreover, both the patient and his wife had contact with several people suffering from upper respiratory tract illness with cough as their main symptom, during festivities for the Greek National day on 25 March, four days prior to illness onset for the wife and 14 days prior to illness onset for the patient and during one outdoor dinner on 27 March.

\section{Case definition}

In Greece a possible case of MERS-CoV infection is defined as follows:

Any patient with an acute respiratory infection, that may include history of fever $\left(\geq 38{ }^{\circ} \mathrm{C}\right)$ and cough and indications of pulmonary parenchymal disease (i.e. clinical, radiological or histopathological evidence of pneumonia or acute respiratory distress syndrome (ARDS)), AND at least one of the following:

- history of travel to or residence in affected areas (in the Middle East), during the 14 days before symptom onset;

- close contact, during the 14 days before symptom onset, with a symptomatic confirmed case of MERS-CoV infection;

\section{FIGURE}

Timeline of possible exposure and clinical course of Middle East Respiratory Syndrome coronavirus infection case, Greece March-April 2014

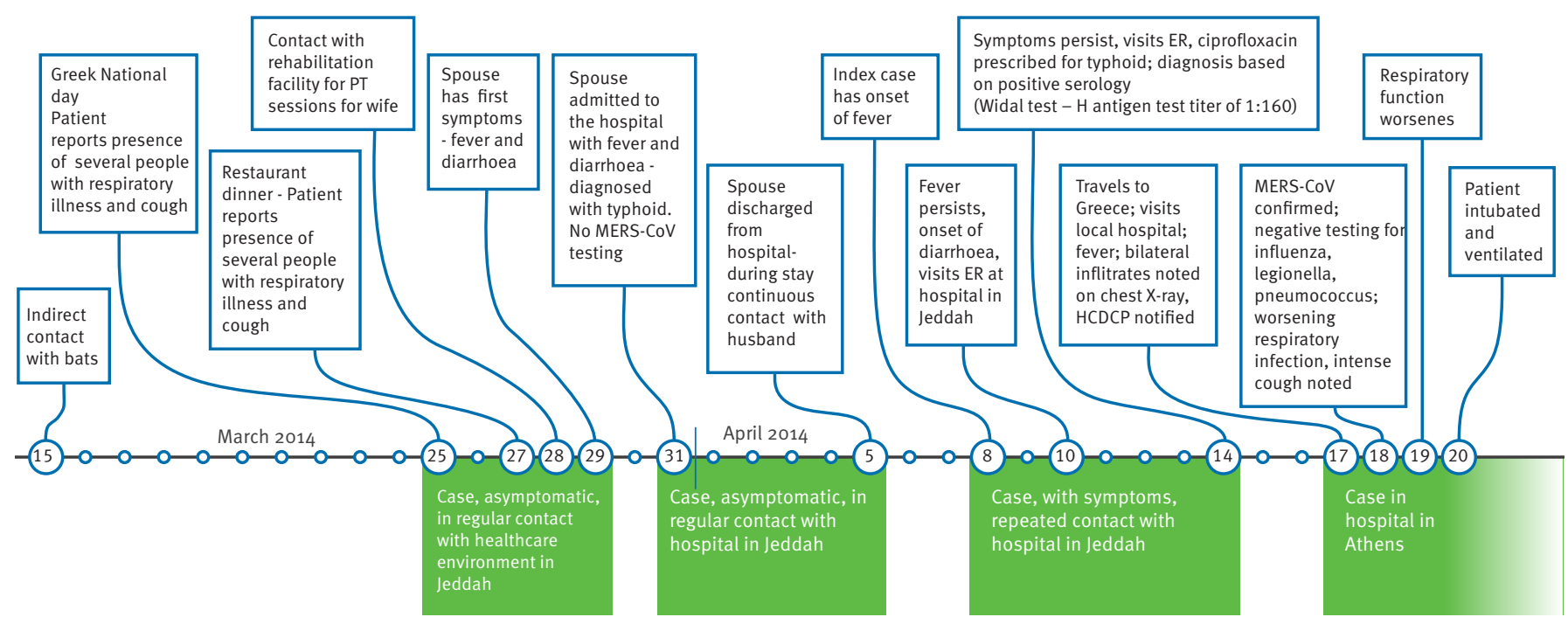

ER: emergency room; HCDCP: Hellenic Center for Disease Control and Prevention; MERS-CoV: Middle East Respiratory Syndrome coronavirus; PT: physiotherapy 
- being a healthcare worker that cared for a possible or confirmed case of severe respiratory infection attributed to the new coronavirus;

- belonging to a cluster of severe lower respiratory tract respiratory infections;

- respiratory illness not responding to therapeutic measures instituted by the treating physician.

A probable case of MERS-COV infection in Greece is defined as follows:

A person with a febrile acute respiratory illness with clinical, radiological or histopathological evidence of pulmonary parenchymal disease (e.g. pneumonia or ARDS), AND

- for whom MERS-CoV infection has not been laboratory confirmed, AND

- who has a direct epidemiological link to a confirmed MERS-CoV case.

A confirmed case of MERS-COV is defined as a person with laboratory confirmation of MERS-CoV infection.

According to the Hellenic Center for Disease Control and Prevention (HCDCP) guidelines, clinical samples from the upper respiratory tract (nasopharyngeal swabs), as well as lower respiratory tract specimens (sputum or bronchoalveolar lavage fluid, when possible) have to be collected and tested for MERS-CoV in an initial screening by one of the National Reference Laboratories (Hellenic Pasteur Institute, Microbiology Department University of Athens Medical School, Microbiology Department Aristotle University of Thessaloniki Medical School).

\section{Contact tracing}

As soon as the diagnosis was confirmed, contact tracing was initiated by the national public health authorities. Despite the reported lack of respiratory symptoms in the patient, Greek authorities decided to aggressively trace all contacts since: (i) the exact mode of transmission of the MERS-CoV virus is not known; (ii) the patient had fever and diarrhoea and; (iii) he was not continuously using his face mask and there was the possibility that he had mild respiratory symptoms that he ignored.

Contacts were defined according to the scheme proposed in a previous publication [6] as all people who either had close contact with the confirmed case during (i) the air travel, (ii) his 'community stay' between the landing of the airplane and his visit to the hospital, (community contacts were defined as i.e. contact with the patient at the household setting, any person who had prolonged (>15 minutes) face-to-face contact with the confirmed case any time during the illness in a household setting or in any other enclosed setting), or (iii) his hospital stay (i.e. a healthcare worker (HCW) who provided direct clinical or personal care to, or examined the symptomatic confirmed case, or was within close vicinity of an aerosol-generating procedure). HCW contacts were further sub-classified according to whether they wore full personal protective equipment at the time of contact i.e. correctly fitted high filtration mask, gown, gloves and eye protection or not.

Surveillance of contacts

Active follow-up of all cases was initiated by the command center of the HCDCP for all outpatient contacts and from the hospital infection control committee for all HCW. Contacts were contacted by phone and were asked to report to $\mathrm{HCDCP}$ any fever $\geq 38^{\circ} \mathrm{C}$ (via regular temperature checking preferably twice a day) and/ or respiratory tract symptoms and/or digestive tract symptoms during a 14-day period, equal to the maximum incubation period for MERS-CoV according to WHO guidance [7], after their last contact with the confirmed case. Contacts were also provided with a hotline number (the number of the HCDCP command center which operates on a $24 / 7$ basis) and instructed to call anytime in case of any symptoms or other questions.

For all contacts voluntary baseline and follow-up (15 days) serological sampling was offered for future testing for MERS-CoV antibodies. Oropharyngeal swabbing was offered free of charge to all contacts especially those experiencing symptoms as well as immediate prolonged face-to-face contact with the case.

Results from contract tracing

Seventy-three contacts were identified and placed under clinical surveillance; 12 from the two flights i.e. passengers who were on the same flight as the MERSCoV case with an assigned seat in the same row and in the two rows in front and behind him, nine community contacts and $52 \mathrm{HCW}$ at the two hospitals (six not using personal protective equipment). Prolonged faceto-face contact with the index case, defined as duration of at least 15 minutes within one meter from the confirmed case, was identified in two HCW.

Five contacts chose to undergo oropharyngeal swab testing, two relatives, one HCW and two people exhibiting mild respiratory or gastrointestinal symptoms within three days after contact. All tested negative for MERS-CoV and were offered repeated testing after seven days or earlier if symptoms worsened.

\section{Further public health measures}

Revised guidance to HCW for early recognition and response including the institution of the necessary screening, prevention and infection control measures for all suspected cases presenting to Greek Hospitals was sent by email to all hospital administrations to be further distributed to the Infection Control Committee of each hospital and from there to all HCWs, on 19 April. A triage procedure for MERS-CoV using clinical and epidemiological criteria is recommended in all Greek hospitals according to the existing national guidance [8]. 
The HCDCP travel office has issued extensive guidance for points of entry and especially airports, regarding information to all outgoing and incoming travelers from affected areas, as well as guidance for healthcare services at the airport and air flight crew members. Special posters and leaflets have been distributed throughout the main airports of the country, especially in Athens.

The HCDCP is in continuous communication with the Greek Foreign Ministry, Ministry of Defense, the Civil Aviation service, and consulate offices in Saudi Arabia. Appropriate communication regarding the case was initiated at the European level, though the European Early Warning and Response System (EWRS) on 18 April 2014. In addition, the International Health Regulation (IHR) focal points in Saudi Arabia and Jordan were notified about the case and possible exposure of people in their countries.

A press release was issued on 18 April 2014 by the $\mathrm{HCDCP}$ to inform the general public about MERS-CoV and preventive measures.

\section{Discussion}

As of 20 April 2014, 250 laboratory confirmed cases of MERS-CoV have been confirmed worldwide since the first detection of this novel virus in Saudi Arabia, including 93 deaths [9]. Herein, we report the fourth imported and tenth laboratory confirmed case in an otherwise healthy adult, in the European Union. Other cases have been reported in the United Kingdom, Germany, France and Italy [10-13].

The source of infection of our patient although unclear, is most likely respiratory transmission from a patient, in the healthcare environment, in Saudi Arabia, as previously reported for other cases [14-17]. Backward investigation revealed close contact with the healthcare environment in Jeddah over several weeks. Regarding other possible sources of infection we doubt that his brief exposure to bats during dinner with friends in a neighborhood of Jeddah, outside the incubation period for MERS-CoV could have constituted a significant risk for infection. However, a zoonotic hypothesis should be always considered in similar cases: because the patient did not report any direct contact with bats or camels, this does not mean he did not have any exposure to an animal source of virus that he might have forgotten or not been aware of.

The information about several cases of respiratory illness in the community, deserves attention and should be further investigated. Both the patient and his wife described extensive spread of a 'respiratory illness' in the community during the incubation period. Alternatively, he could have contracted the infection from his wife if her febrile diarrhoeal episode was associated with MERS-CoV. Although she tested negative for MERS-CoV, she did not have an x-ray, furthermore she could have stopped shedding virus by the time she arrived to Greece and only serological testing will prove whether she had a past infection. The National Reference Center will perform serological tests as soon as an assay becomes available locally. Family clusters of MERS-CoV infections have been described even with asymptomatic or mild cases [18]. If the patient's wife would have been the source of infection, then she would also be a case without respiratory signs and with a very short shedding period; however, this is highly improbable unless she also is a secondary case. If not acquired from his wife, the infection could have been acquired during one of the patient's visits on 10 or 14 April at the local hospital in Jeddah. At this moment, we do not have any official confirmation if there had been cases of MERS-CoV in the hospital.

We are not fully convinced by the diagnosis of typhoid fever in this patient because of the negative laboratory findings despite continued diarrhoea, upon presentation in Greece and the low specificity of a single Widal test [19]. Even though the diarrhoea could have been due to some persistent bowel irritation from a previous typhoid episode, we rather believe that his diarrhoea might be related to the MERS-CoV infection.

Our case together with another recent case of MERS$\mathrm{CoV}$ infection in a traveler from Jeddah to Malaysia [20] as well as the increasing number of newly reported cases by the Ministry of Health of Saudi Arabia in the previous days [21], highlight the need for enhanced awareness regarding the presence of the virus in (i) all persons with fever especially when hospitalised in Saudi Arabia, possibly even when only gastrointestinal symptoms are present and in (ii) travelers coming from the Arabian Peninsula with symptoms compatible with MERS-CoV infection even when atypical, especially if fever is present. In support of the latter we like to point out that our patient had a negative chest $\mathrm{x}$-ray during his hospital evaluation in Saudi Arabia. Moreover, we emphasise the need for vigilance and institution of appropriate, strict infection control measures at the hospital environment in Saudi Arabia.

The absence of respiratory symptoms early in the course of his disease is puzzling in our case. The possibility of mild respiratory symptoms gone unnoticed exists. Thus, we used a rather sensitive case definition for close contacts that included diarrhoea in order to identify new infections presenting in a similar manner to the index patient and prevent further spread.

Since large numbers of Greeks reside in the Arabian peninsula and there is ongoing travel to the affected areas, Greek authorities are considering to issue specific guidance to avoid travel for travelers at risk for developing severe disease similar to the one issued by the Saudi Arabia Ministry of Health for the pilgrimages of Umrah and Hajj [22]. European level action to this effect is anxiously awaited.

In conclusion, every new MERS-CoV case, independent of whether it occurs in the Arabian peninsula, Europe 
or elsewhere, and the response to it, is associated with a high work load and investment of resources for the public health sector. However, it is at the same time a step forward and an opportunity to realise the gaps in knowledge associated with this relatively new worldwide threat.

\section{Acknowledgments}

The authors are grateful to all the authorities and HCW involved in the response to the case. They extend special thanks to the health authorities and civil airport service of the Eleftherios Venizelos Airport that assisted with contact tracing activities.

\section{Conflict of Interest}

None declared

\section{Authors' contributions}

Sotirios Tsiodras together with George Saroglou, Panos Efstathiou, Athanassios Tsakris, Jenny Kremastinou and Theodoros Papadimitriou coordinated the response to the case at the national level and at the two hospitals and wrote the first draft of manuscript together with Agoritsa Baka, Dimitrios Eliopoulos and Andreas Mentis.

Sotirios Tsiodras, Agoritsa Baka, Dimitris Eliopoulos, Xanthi Dedoukou, Georgia Papamavrou, Spyridoula Karadima, Androula Pavli, Georgia Spala and Helena Maltezou, performed the contact tracing activities.

Maria Emmanouil, Thanos Kossyvakis, Nikolaos Spanakis, Vasiliki Pitiriga, Andreas Mentis, and Athanassios Tsakris performed the laboratory investigation.

Aikaterini Karageorgou and Panos Efstathiou, coordinated the response at the hospital level both locally and nationally

Sotirios Tsiodras, Epaninodnas Kosmas, Stavros Tsiagklis, Spyridon Gkatzias, Nikolaos G. Koulouris, Antonia Koutsoukou, Petros Bakakos, Evangelos, Markozanhs, George Dionelis, Kostantinos Pontikis, Nikoletta Rovina, Magdalene Kyriakopoulou and George Saroglou provided care for the patient.

All co-authors critically read and revised the drafts of the manuscript.

\section{* Authors' correction:}

On request of the authors, the words 'in an oropharyngeal sample' were added to the sentence "On 18 April, the MERSCoV infection was confirmed, in an oropharyngeal sample, based on real-time RT- PCR using primers for the upstream of envelope gene (UPE) as a screening test and for the open reading frame (ORF) $1 \mathrm{~A}$ gene as a confirmatory assay [1]." and the following sentence was deleted: "Tests for MERSCoV using real-time RT-PCR in an oropharyngeal and a rectal swab as well as in a blood and a urine sample were negative". This correction was made on 28 April.

\section{References}

1. Corman VM, Muller MA, Costabel U, Timm J, Binger T, Meyer $B$, et al. Assays for laboratory confirmation of novel human coronavirus (hCoV-EMC) infections. Assays for laboratory confirmation of novel human coronavirus (hCoV-EMC) infections. Euro Surveill. 2012;17(49): pii=20334.

2. World Health Organization (WHO). Middle East Respiratory Syndrome Coronavirus (MERS-CoV). Initial Interview Questionnaire of Cases. World Health Organization. Available from: http://www.who.int/csr/disease/coronavirus_infections/ MERS_case_investigation_questionnaire.pdf

3. Meyer B, Muller MA, Corman VM, Reusken CB, Ritz D, Godeke GJ, Lattwein E, et al. Antibodies against MERS Coronavirus in Dromedary Camels, United Arab Emirates, 2003 and 2013. Emerg Infect Dis. 2014;20(4):552-9. http://dx.doi.org/10.3201/eid2004.131746

4. Woo PC, Lau SK, Wernery U, Wong EY, Tsang AK, Johnson B, et al. Novel betacoronavirus in dromedaries of the middle East, 2013. Emerg Infect Dis. 2014;20(4):560-72. http://dx.doi.org/10.3201/eid2004.131769

5. Memish ZA, Mishra N, Olival KJ, Fagbo SF, Kapoor V, Epstein JH, et al. Middle East respiratory syndrome coronavirus in bats, Saudi Arabia. Emerg Infect Dis. 2013;19(11):1819-23. http://dx.doi.org/10.3201/eid1911.131172

6. The Health Protection Agency (HPA) UK Novel Coronavirus Investigation team. Evidence of person-to-person transmission within a family cluster of novel coronavirus infections, United Kingdom, February 2013 . Euro Surveill. 2013;18(11): pii=20427.

7. World Health Organization (WHO). WHO guidelines for investigation of cases of human infection with Middle East Respiratory Syndrome Coronavirus (MERS-CoV). Available from: http://www.who.int/csr/disease/coronavirus infections/ MERS_CoV_investigation_guideline_Jul13.pdf

8. Hellenic Center for Disease Control and Prevention (HCDCP). Guidance for handling suspect cases of MERS-Coronavirus infection. Athens: HCDCP; Hellenic Center for Disease Control and Prevention. [Accessed 23 April 2014]. Available from: http://www.keelpno.gr/Portals/o/\%CE\%91\%CF\% $81 \%$ CF\% $87 \%$ CE\%B $5 \%$ CE\%AF\%CE\%B1/\%CE\% $91 \%$ CE\%BD\%CE\%B1\%CF\% 80 $\%$ CE $\%$ BD $\%$ CE $\%$ B $5 \%$ CF $\% 85 \%$ CF\% $83 \%$ CF\% $84 \%$ CE\%B9\%CE\%BA $\%$ CE\%BF\%CF\%8D/Coronavirus_new/CORONAvirus-1_April\%20 2014.pdf

9. World Health Organization (WHO). Middle East respiratory syndrome coronavirus (MERS-CoV) - update. Disease Outbreak News. Available from: http://www.who.int/csr/ don/2014_04_20_mers/en/

10. Bermingham A, Chand MA, Brown CS, Aarons E, Tong C, Langrish C, et al. Severe respiratory illness caused by a novel coronavirus, in a patient transferred to the United Kingdom from the Middle East, September 2012. Euro Surveill. 2012;17(40): pii=20290.

11. Buchholz U, Muller MA, Nitsche A, Sanewski A, Wevering N, Bauer-Balci T, et al. Contact investigation of a case of human novel coronavirus infection treated in a German hospital, October-November 2012.

Euro Surveill. 2013;18(8). pii: 20406.

12. Mailles A, Blanckaert K, Chaud P, van der Werf S, Lina B, Caro V, et al. First cases of Middle East Respiratory Syndrome Coronavirus (MERS-CoV) infections in France, investigations and implications for the prevention of humanto-human transmission, France, May 2013. Euro Surveill. 2013;18(24): $\mathrm{pii}=20502$.

13. Puzelli S, Azzi A, Santini MG, Di Martino A, Facchini M, Castrucci MR, et al. Investigation of an imported case of Middle East Respiratory Syndrome Coronavirus (MERS-CoV) infection in Florence, Italy, May to June 2013. Euro Surveill. 2013;18(34): pii=20564.

14. Centers for Disease Control and Prevention (CDC). Update: Severe respiratory illness associated with Middle East Respiratory Syndrome Coronavirus (MERS-CoV)--worldwide, 2012-2013. MMWR Morb Mortal Wkly Rep. 2013;62(23):480-3.

15. Guery B, Poissy J, el Mansouf L, Sejourne C, Ettahar N, Lemaire $X$, et al: Clinical features and viral diagnosis of two cases of infection with Middle East Respiratory Syndrome coronavirus: a report of nosocomial transmission. Lancet. 2013;381(9885):2265-72. http://dx.doi.org/10.1016/S0140-6736(13)60982-4

16. Assiri A, McGeer A, Perl TM, Price CS, Al Rabeeah AA, Cummings DA, et al. Hospital outbreak of Middle East respiratory syndrome coronavirus. N Engl J Med. 2013;369(5):407-16. http://dx.doi.org/10.1056/NEJMoa1306742

17. Memish ZA, Zumla Al, Assiri A. Middle East respiratory syndrome coronavirus infections in health care workers. $\mathrm{N} \mathrm{Engl}$ I Med. 2013 Aug 29;369(9):884-6. http://dx.doi.org/10.1056/NEJMc1308698

18. Omrani AS, Matin MA, Haddad Q, Al-Nakhli D, Memish ZA, Albarrak AM. A family cluster of Middle East Respiratory Syndrome Coronavirus infections related to a likely unrecognized asymptomatic or mild case. Int J Infect Dis. 
2013;17(9):e668-72

http://dx.doi.org/10.1016/j.ijid.2013.07.001

19. Keddy KH, Sooka A, Letsoalo ME, Hoyland G, Chaignat CL, Morrissey AB,et al. Sensitivity and specificity of typhoid fever rapid antibody tests for laboratory diagnosis at two sub-Saharan African sites. Bull World Health Organ. 2011;89(9):640-7.

http://dx.doi.org/10.2471/BLT.11.087627

20. Middle East respiratory syndrome coronavirus (MERS-

(oV) - update. Disease Outbreak News by the World Health

Organization. [Accessed 23 April 2014]. Available from: http://

www.who.int/csr/don/2014_04_17_mers/en/

21. Middle East respiratory syndrome coronavirus (MERS-

CoV) - update. Disease Outbreak News by the World Health Organization. Available from: http://www.who.int/csr/ don/2014_04_14_mers/en/

22. Ministry of Health, Saudi Arabia. $\mathrm{MOH}$ Issues Health Regulations for Those Flocking to Saudi Arabia to Perform Umrah and Hajj. [Accessed 23 April 2014]. Available from: http://www.moh.gov.sa/en/CoronaNew/News/Pages/news2013-7-14-001.aspx 